\title{
Fluid secretion in epithelial cells: a key role of the Na-k-2cl cotransporter I
}

\author{
Abbreviations: NKCC1, Na-k-2cl cotransporter isoform 1; \\ $\mathrm{PKC}$, protein kinase $\mathrm{c}$
}

\section{Editorial}

In epithelial cells such as those lining the colon or the upper airway, fluid secretion serves to lubricate and protect the surface of the epithelia. Dysfunction of fluid secretion is the hallmark of several human diseases (e.g., secretory diarrhea, cystic fibrosis or Sjogren's syndrome). In secretory epithelia, fluid secretion is linked to transepithelial anions secretion (e.g., chloride or bicarbonate). For many years investigators have tried to manipulate anion secretion to treat fluid-associated disorders but with minimal success.

Although most of what we know about regulation of chloride secretion in epithelia derives from work on apical chloride channels (e.g., cystic fibrosis transmembrane regulator, bestrophin or TMEM16), the basolateral Na-K-2Cl cotransporter 1 (NKCC1) is the main chloride loading mechanism-bringing chloride above its electrochemical gradient for secretion by the apical chloride channels. Recent work on the regulation of NKCC1 demonstrates new pathways to control chloride secretion.

Dynamic regulation of plasma membrane proteins availability through endo/exocytosis and recycling represents a powerful mechanism for fine tuning cellular response (e.g., ion transport, hormone response). In colonic epithelial cells, activation of novel $\mathrm{PKC}$ delta $(\mathrm{PKC} \delta)$ and epsilon (PKC $)$, but not the conventional PKC alpha, are responsible for the rapid internalization of NKCC1. Activation of $\mathrm{PKC} \varepsilon$ causes a more profound inhibition of chloride secretion than $\mathrm{PKC} \delta$ activation, demonstrating that chloride secretion can be fine-tuned according to the PKC isoform activated. ${ }^{1}$ Furthermore, NKCC1 internalization during PKC activation is clathrin-mediated which implies that $\mathrm{NKCC1}$ is either subject to degradation or recycling. ${ }^{2}$ This finding has major consequences on the chloride secretion. Degradation of NKCC1 would imply prolong refractory period in chloride secretion, whereas recycling would provide a mean for rapid mobilization of $\mathrm{NKCC1}$ and reestablishment of chloride secretion. Capsaicin, the pungent ingredient of hot pepper, also blunts chloride secretion by causing internalization of NKCC1 through an independent pathway of its usual target the Transient Receptor Potential Vanilloid type $1 .^{3}$ Aside from the regulation of NKCC1 membrane expression, kinases are involved in the control of NKCC1 activity. ${ }^{4-6}$

Sustaining chloride secretion by preventing NKCC1 internalization may dramatically improve chloride secretion in cystic fibrosis during activation of other chloride channels or in settings aiming to rescue cystic fibrosis transmembrane regulator trafficking. On the other hand, activating pathways leading to NKCC1 internalization may prove to be useful drugs against secretory diarrhea. Therefore, understanding
Volume I Issue 2 - 2014

\author{
Patrice G Bouyer \\ Department of Biology, Valparaiso University, USA
}

Correspondence: Patrice G Bouyer, Valparaiso University, Department of Biology, Neils Science, Center, Rm I05A, 1610 Campus Drive East,Valparaiso, IN 46383 USA, Tel 2194645487, Fax 2194645489,Email patrice.bouyer@valpo.edu

Received: June 23, 2014 | Published: June 24, 2014

regulation of chloride secretion by NKCC1 may have important therapeutic applications.

\section{Acknowledgements}

None.

\section{Conflict of interest}

The author declares no conflict of interest.

\section{References}

1. Tang J, Bouyer P, Mykoniatis A, et al. Activated PKC $\{$ delta $\}$ and PK$\mathrm{C}\{$ epsilon\} inhibit epithelial chloride secretion response to cAMP via inducing internalization of the $\mathrm{Na}+-\mathrm{K}+-2 \mathrm{Cl}-$ cotransporter $\mathrm{NKCC} 1 . J$ Biol Chem. 2010;285(44):34072-34085.

2. Mykoniatis A, Shen L, Fedor-Chaiken M, et al. Phorbol 12-myristate 13-acetate-induced endocytosis of the $\mathrm{Na}-\mathrm{K}-2 \mathrm{Cl}$ cotransporter in MDCK cells is associated with a clathrin-dependent pathway. Am J Physiol Cell Physiol. 2010;298:C85-C97.

3. Bouyer PG, Tang X, Weber CR, et al. Capsaicin induces NKCC1 internalization and inhibits chloride secretion in colonic epithelial cells independently of TRPV1. Am J Physiol Gastrointest Liver Physiol. 2013;304(2):G142-G156.

4. Darman RB, Forbush B. A regulatory locus of phosphorylation in the $\mathrm{N}$ terminus of the Na-K-Cl cotransporter, NKCC1. J Biol Chem. 2002;277(40):37542-37550.

5. Gagnon KB, England R, Delpire E. Volume sensitivity of cation-Cl- cotransporters is modulated by the interaction of two kinases:Ste20-related proline-alanine-rich kinase and WNK4. Am J Physiol Cell Physiol. 2006;290:C134-C142.

6. Ponce-Coria J, Markadieu N, Austin T, et al. A Novel Ste20-related Proline/Alanine-rich Kinase (SPAK)-independent Pathway Involving Calcium-binding Protein 39 (Cab39) and Serine Threonine Kinase with No Lysine Member 4 (WNK4) in the Activation of Na-K-Cl Cotransporters. J Biol Chem. 2014;289(25):17680-17688. 